\title{
Economic Determination of Modified Multiple Dependent State Sampling Plan under Some Lifetime Distributions
}

\author{
Muhammad Aslam (D, , ${ }^{1}$ P. Jeyadurga, ${ }^{2}$ S. Balamurali, ${ }^{2}$ Muhammad Azam, ${ }^{3}$ \\ and Ali AL-Marshadi ${ }^{1}$ \\ ${ }^{1}$ Department of Statistics, Faculty of Science, King Abdulaziz University, Jeddah 21551, Saudi Arabia \\ ${ }^{2}$ Department of Computer Applications, Kalasalingam Academy of Research and Education, Krishnankoil, \\ Tamil Nadu 626126, India \\ ${ }^{3}$ Department of Statistics and Computer Science, University of Veterinary and Animal Sciences, Lahore 54000, Pakistan \\ Correspondence should be addressed to Muhammad Aslam; aslam_ravian@hotmail.com
}

Received 3 July 2020; Revised 14 November 2020; Accepted 11 January 2021; Published 29 January 2021

Academic Editor: Markos Koutras

Copyright (c) 2021 Muhammad Aslam et al. This is an open access article distributed under the Creative Commons Attribution License, which permits unrestricted use, distribution, and reproduction in any medium, provided the original work is properly cited.

\begin{abstract}
In this paper, the modification of multiple dependent state sampling plan is proposed and designed for assuring a mean lifetime of the products under Birnbaum-Saunders distribution and Weibull distribution. The optimal parameters of the proposed plan are determined based on two points on the operating characteristic curve approach. Different combinations of producer's risk and consumer's risk are considered for plan parameters determination. The efficacy of the proposed plan is compared with those of other existing sampling plans using an average sample number and operating characteristic function. The economic designing of the proposed plan is also considered and the comparative study is done based on the total cost of the inspection.
\end{abstract}

\section{Introduction}

Acceptance sampling is playing an important role to ensure the quality of the product through proper inspection of the raw material and finished products. Inspection of a lot of products through the acceptance sampling will safeguard the consumers and the producers by showing products' quality to select the products with confidence and by providing a chance to prove the products quality with low cost, respectively. At the time of inspection, a random sample of items selected from the lot is tested and lot-sentencing is done on the basis of information obtained from the sample(s). A lot of products are accepted if the total number of nonconforming items or failures is less than or equal to the allowable number of nonconforming items or failure items or acceptance number.

Due to the high reliability of electronic products, it may not be possible to test all items for ensuring average life of the product. Therefore, the use of an acceptance sampling plan is very helpful in making a decision about the lot before it is supplied to the market. For example, in life testing, a sample of items is selected and put on the test for some specified time. The failure time of the component/product is modeled by some statistical distributions. An acceptance/ rejection decision is made using sample size and allowed number of failure items for the test. The lot of products is accepted if the allowed or lesser number of failures is observed before the inspection time is ended; otherwise, a lot of products are rejected. Several sampling plans are available in the literature; among those, single sampling plan (SSP) is the simplest plan to implement in industries such as electronic products manufacturing. In SSP, a decision about the lotsentencing is taken using single sample information. A number of authors have investigated on designing of acceptance sampling plans for ensuring the lifetime of the products under various lifetime distributions. including $[1,2]$. Jun et al. [1] proposed variables SSP and double sampling plan (DSP) under Weibull distribution and they utilized the concept of sudden death testing in order to reduce the inspection time. In addition, they proved that the 
average sample number (ASN) can be reduced by DSP. In order to reduce the inspection time as well as inspection cost, Rao [2] proposed group acceptance sampling plans under Weibull distribution and generalized exponential distribution, respectively. SSP has been designed under Birnbaum-Saunders distribution and generalized exponential distribution, respectively, by [3]. Rasay et al. [4] considered the designing of a sequential sampling plan under Weibull distribution based on time truncated life test. Obviously, the inspection cost depends on the sample size selected from a lot of the products. Lio et al. [3] have shown that SSP required a larger sample to make an acceptance/rejection decision about the lot. The larger sample size means it consumes more time and cost for the life test experiment. Therefore, some more efficient sampling schemes such as multiple dependent state (MDS) sampling plan can be applied for the reduction of inspection cost and make a decision by utilizing the current sample and/or previous sample information. Wortham and Baker [5] introduced MDS sampling. Balamurali and Jun [6] proved that the MDS sampling plan is more efficient than the SSP in terms of sample size. Balamurali et al. [7] designed an MDS sampling plan to ensure the median life of the products under generalized inverted exponential distribution. Nadi and Gildeh [8] proposed a group MDS sampling plan to assure the actual Weibull distributed mean life of the product, which is longer than the specified one. Aslam et al. [9] studied the generalized MDS sampling plan to provide mean life assurance for the products under three lifetime distributions: gamma distribution, Burr type XII distribution, and Birnbaum-Saunders distribution. More details about such sampling plans can be seen in [10-12].

The MDS sampling plans available in the literature utilize the sample information of current lot and/or preceding lots. Although MDS sampling plan considers certain amount of preceding lots' sample information to dispose the current lot having moderate quality using minimum sample size, this plan calls for immediate rejection of the current lot if any of the preceding lots has moderate quality even when remaining lots are of good quality (i.e., any of the samples from the specified number of preceding lots' consists of failure items such as $c_{1}<d \leq c_{2}$ ). This will definitely increase the producer's risk. Hence, there is a necessity to introduce a new sampling plan to overcome the aforementioned drawback of MDS sampling plan. For this reason, in this paper, we introduce a modified version of the MDS sampling plan to achieve more reduction in the sample size with the desired protection. It is expected that the modified MDS sampling plan will be more flexible and more efficient than the existing MDS sampling plan in terms of sample size and cost required for the inspection. By exploring the literature and to the best of our knowledge, there is no study on the design of sampling plans for life tests using the modified MDS sampling. In this paper, we attempt to design this sampling plan for some popular distributions. We will also develop a cost model for the modified MDS sampling plan. The comparison of the proposed plan with existing plans is given in terms of cost and sample size.

\section{Weibull and Birnbaum- Saunders Distributions}

2.1. Weibull Distribution. The Weibull distribution has been applied in numerous areas to model the time to failure of the product and also this distribution is considered as a suitable model for failure forecasting and prediction (see [13]). In engineering fields, this distribution is used to fit the strength reliability of components. In biology, the survival analysis (see [14]) can be done under Weibull distribution. In acceptance sampling, this distribution plays an important role when designing the sampling plan for providing a product's lifetime assurance. Nowadays, the Weibull distribution is used in statistical quality control (SQC) to design the control chart and to design the sampling plan (see, e.g., [1, 15-17]). In this paper, we design a modified MDS sampling plan under Weibull distribution for providing mean lifetime assurance for the product. Let us assume that the time to failure of a product follows a Weibull distribution and the cumulative distribution function $(\mathrm{CDF})$ is given as follows:

$$
F(t ; \lambda, \delta)=1-\exp \left(-\left(\frac{t}{\lambda}\right)^{\delta}\right), \quad t \geq 0, \lambda>0, \delta>0,
$$

where $\delta$ is the known shape parameter and $\lambda$ is an unknown scale parameter. The mean of the Weibull distribution is obtained as

$$
\mu=\left(\frac{\lambda}{\delta}\right) \Gamma\left(\frac{1}{\delta}\right)
$$

where $\Gamma(\cdot)$ is the complete gamma function. The probability that the product will fail before the experiment time $t_{0}$ under the Weibull distribution is obtained by the following equation:

$$
p=1-\exp \left(-\left(\frac{t_{0}}{\lambda}\right)^{\delta}\right)
$$

We write the experiment time $t_{0}$ in terms of specified mean life $\mu_{0}$ such as $t_{0=} a \mu_{0}$ for a constant $a$ (experiment termination ratio). Therefore, the equation of obtaining the failure probability of a product before the experiment time $t_{0}$ under the Weibull distribution can be rewritten as

$$
p=1-\exp \left(-a^{\delta}\left(\frac{\mu_{0}}{\mu}\right)^{\delta}\left(\frac{\Gamma(1 / \delta)}{\delta}\right)^{\delta}\right)
$$

Sometimes, the shape parameter of the product lifetime distribution is unknown and such situations are handled by estimating the unknown shape parameter using the past history of product lifetime data. For any specified values of the shape parameter, mean ratio, and experiment termination ratio, the failure probability can be calculated using equation (4).

2.2. Birnbaum-Saunders Distribution. The Birnbaum-Saunders (BS) distribution was introduced by [18] to model the failures due to cracks. This distribution is also known as the fatigue life distribution and is extensively used in reliability 
studies. This distribution has been used in various fields including electronics and food industry and medical and construction fields. For example, [19] applied the process capability indices for BS processes in the electronics and food industries. Desousa et al. [20] utilized this distribution and they have shown that this distribution is a good alternative to describe the medical data. Leiva et al. [21] used BS distribution to model the hardness of polymeric bone cement. In addition, this distribution and generalized form of this distribution have been applied in SQC to design the sampling plan as well as the control chart (see, i.e., [22, 23]). In this paper, we use the BS distribution to design a modified MDS sampling plan for ensuring the product's mean lifetime. The CDF of BS distribution is

$$
F(t ; \omega, \sigma)=\Phi\left[\frac{1}{\omega}\left\{\left(\frac{t}{\sigma}\right)^{(1 / 2)}-\left(\frac{\sigma}{t}\right)^{(1 / 2)}\right\}\right], t \geq 0, \omega>0, \sigma>0,
$$

where $\omega$ is the shape parameter and it is assumed to be known, $\sigma$ is the unknown scale parameter, and $\Phi(\cdot)$ is a standard normal CDF. The mean of the BS distribution is given as follows:

$$
\mu=\sigma\left(1+\frac{\omega^{2}}{2}\right)
$$

The failure probability of the product before the experiment time $t_{0}$ under BS distribution is

$$
p=\Phi\left[\frac{1}{\omega}\left\{\left(\frac{t_{0}}{\sigma}\right)^{(1 / 2)}-\left(\frac{\sigma}{t_{0}}\right)^{(1 / 2)}\right\}\right]
$$

We can write the scale parameter in terms of mean value and the experiment time $t_{0}$ is expressed as a product of experiment termination ratio and specified mean life $\mu_{0}$ such as $t_{0} a \mu_{0}$. Therefore, the failure probability of a product before the experiment time $t_{0}$ where the lifetime follows BS distribution is given as

$$
p=\Phi\left[\frac{1}{\omega}\left\{\left(\frac{a\left(1+\left(\omega^{2} / 2\right)\right)}{\left(\mu / \mu_{0}\right)}\right)^{(1 / 2)}-\left(\frac{\left(\mu / \mu_{0}\right)}{a\left(1+\left(\omega^{2} / 2\right)\right)}\right)^{(1 / 2)}\right\}\right]
$$

The above equation can be used to obtain the failure probability of the product for different combinations of the shape parameter, mean ratio, and experiment termination ratio, where the lifetime follows BS distribution. In this design, the sample item is classified as nonconforming if it fails before the experiment time $t_{0}$.

\section{Designing of Modified MDS Sampling Plan Based on Time Truncated Life Test}

In the literature of acceptance sampling, to overcome the drawbacks of zero acceptance number SSP, Dodge (1955) introduced a sampling plan called chain sampling plan and designated as ChSP-1. In 1976, the generalized version of ChSP1 was introduced by Wortham and Baker [5] and named as an MDS sampling plan. These two sampling plans are also categorized under special purpose sampling plans. Many authors have investigated the performance of special purpose sampling plans including ChSP-1 and MDS sampling plan. It has been proved that the reduction of sample size with desired protection for both producer and consumer can be achieved by using the special purpose sampling plans. MDS sampling plan is used to inspect the submitted lots which are coming serially in the order of production and such plans will reduce the sample size rather than conventional sampling plans such as SSP and DSP. Therefore, in this paper, we consider the designing of a modified version of the MDS sampling plan to achieve more reduction in the sample size with the desired protection. The modified MDS sampling plan is also portrayed by four parameters as in MDS sampling plan, namely, $n, c_{1}, c_{2}$, and $m$, where $n$ is the sample size, $c_{1}$ is the maximum number of nonconforming items or failure items for unconditional acceptance $c_{1} \geq 0, c_{2}$ is the maximum number of additional nonconforming items or failure items for conditional acceptance $c_{2}>c_{1}$, and $m$ is the number of preceding lots required for current lot disposition. However, there is a difference between the operating procedures of the MDS sampling plan and the modified MDS sampling plan. That is, both sampling plans provide an additional chance to the producer when the current lot quality is moderate. The difference is that the MDS sampling plan will accept the current lot if preceding " $m$ " samples (i.e., samples from preceding " $m$ " lots) consist of nonconforming items less than or equal to $c_{1}$. In the modified MDS sampling plan, the acceptance of the current lot will be allowed even if any of the " $m$ " preceding lots has moderate quality but the remaining $(m-1)$ samples out of $m$ samples should satisfy the condition as in the MDS sampling plan. The conditions under which the modified MDS sampling plan can be implemented and the operating procedure based on time truncated life test are as follows.

3.1. Conditions for Applications. The development and application of modified MDS sampling plan are the same as in MDS sampling procedures; those are listed as follows: (1) The product to be inspected comprises a series of successive lots produced by a continuing process. (2) Normally, lots are expected to be of essentially the same quality. (3) A fixed number of sample items are selected from each lot. (4) The consumer has faith in the integrity of the producer.

\subsection{Operating Procedure.}

Step 1. Select a random sample of size $n$ from the current lot and perform life test on the sample items for specified time $t_{0}$ and count the number of items that failed before the time $t_{0}$ (or count the number of nonconforming items in the case of binomial distribution); denote it as $d$.

Step 2. Accept the current lot if $d \leq c_{1}$ and reject the current lot if $d>c_{2}$. Otherwise, go to Step 3 .

Step 3. Accept the current lot if there is at most one preceding lot that has the moderate quality and the remaining $(m-1)$ lots out of $m$ preceding lots have been accepted with $d \leq c_{1}$. 
It is obvious that the attributes MDS sampling plan is the general case of SSP. Similarly, it should be noted that the proposed modified MDS sampling plan also converges to SSP under some conditions which are listed as follows:

(i) When $m \longrightarrow \infty$ and/or $c_{2}=c_{1}=c$ (say), modified MDS sampling plan reduces to SSP with acceptance number $c_{1}$.

(ii) When $m=0$, the modified MDS plan reduces to SSP with acceptance number $c_{2}$.

The probability that the acceptance of current lot is based on a single sample is denoted by $L_{1}(p)$ and given as follows:

$$
L_{1}(p)=P\left(d \leq c_{1}\right) .
$$

The probability that the quality of the current lot will be moderate is denoted by $L_{2}(p)$ and obtained as follows:

$$
L_{2}(p)=P\left(c_{1}<d \leq c_{2}\right) .
$$

The probability of acceptance of preceding " $m$ " lots with the condition of $d \leq c_{1}$ is $\left(P\left(d \leq c_{1}\right)\right)^{m}$. Similarly, the probability that any of the preceding " $m$ " lots has moderate quality is $\left\{m\left(P\left(d \leq c_{1}\right)\right)^{m-1}\left(P\left(c_{1}<d \leq c_{2}\right)\right)\right\}$ and also these two events are mutually exclusive. Based on the information of preceding " $m$ " lots, probability of acceptance of the current lot with moderate quality is $\left(P\left(c_{1}<d \leq c_{2}\right)\right)\left[\left(P\left(d \leq c_{1}\right)\right)^{m}+\left\{m\left(P\left(d \leq c_{1}\right)\right)\right.\right.$ $\left.\left.{ }^{m-1}\left(P\left(c_{1}<d \leq c_{2}\right)\right)\right\}\right]$.

Therefore, the operating characteristic (OC) function of modified MDS sampling plan is given by

$$
\begin{aligned}
P_{a}(p)= & P\left(d \leq c_{1}\right)+P\left(c_{1}<d \leq c_{2}\right) \\
& \cdot\left\{\left(P\left(d \leq c_{1}\right)\right)^{m}+m\left(P\left(d \leq c_{1}\right)\right)^{m-1} P\left(c_{1}<d \leq c_{2}\right)\right\} .
\end{aligned}
$$

Under the binomial distribution, equation (11) can be rewritten as

$$
\begin{aligned}
P_{a}(p)= & \sum_{d=0}^{c_{1}}\left(\begin{array}{l}
n \\
d
\end{array}\right) p^{d}(1-p)^{n-d}+\sum_{d=c_{1}+1}^{c_{2}}\left(\begin{array}{l}
n \\
d
\end{array}\right) p^{d}(1-p)^{n-d} \\
& \cdot\left\{\left(\sum_{d=0}^{c_{1}}\left(\begin{array}{l}
n \\
d
\end{array}\right) p^{d}(1-p)^{n-d}\right)^{m}+m\left(\sum_{d=0}^{c_{1}}\left(\begin{array}{l}
n \\
d
\end{array}\right) p^{d}(1-p)^{n-d}\right)^{m-1}\left(\sum_{d=c_{1}+1}^{c_{2}}\left(\begin{array}{l}
n \\
d
\end{array}\right) p^{d}(1-p)^{n-d}\right)\right\}
\end{aligned}
$$

The plan that reduces the inspection time and cost is admirable and is called an economical sampling plan. One can attain such a reduction in time and cost by minimizing ASN. Hence, the main objective of this paper is to reduce the ASN of the modified MDS sampling plan rather than the ASN of the MDS sampling plan. Because of ASN reduction, the proposed plan will be implemented with minimum cost. It is important to note that the ASN of a modified MDS sampling plan is its sample size. When designing the sampling plan for assuring the mean lifetime, the ratio between true mean lifetime and the specified lifetime of the product $\mu / \mu_{0}$ plays an important role. The probability of failure corresponding to the mean ratio is considered as the quality of the products. In this designing, we consider the probabilities of failure corresponding to the mean ratios $\mu / \mu_{0}=2,4,6,8,10$ as acceptable quality level (AQL or $p_{1}$ ) and the probability of failure when the true mean life and the specified mean life are equal (i.e., $\mu / \mu_{0}=1$ ) is considered as limiting quality level (LQL or $p_{2}$ ). We select two points on the OC curve approach to design the sampling plan that considers both producer's and consumer's risks simultaneously. In order to determine the optimal parameters, we use a nonlinear optimization problem in which the minimization of ASN at $p_{1}$ is considered as objective function and the constraints are related to the probabilities of acceptance of the lot at AQL and LQL. The optimization problem is as follows.

Minimize $\operatorname{ASN}\left(p_{1}\right)$.

Subject to $P_{a}\left(p_{1}\right) \geq 1-\alpha$,

$$
P_{a}\left(p_{2}\right) \leq \beta, \quad n>1, m \geq 1, c_{2}>c_{1} \geq 0,
$$

where,

$$
\begin{aligned}
P_{a}\left(p_{1}\right)= & \sum_{d=0}^{c_{1}}\left(\begin{array}{l}
n \\
d
\end{array}\right) p_{1}^{d}\left(1-p_{1}\right)^{n-d}+\sum_{d=c_{1}+1}^{c_{2}}\left(\begin{array}{l}
n \\
d
\end{array}\right) p_{1}^{d}\left(1-p_{1}\right)^{n-d} \\
& \cdot\left\{\left(\sum_{d=0}^{c_{1}}\left(\begin{array}{l}
n \\
d
\end{array}\right) p_{1}^{d}\left(1-p_{1}\right)^{n-d}\right)^{m}+m\left(\sum_{d=0}^{c_{1}}\left(\begin{array}{l}
n \\
d
\end{array}\right) p_{1}^{d}\left(1-p_{1}\right)^{n-d}\right)^{m-1}\left(\sum_{d=c_{1}+1}^{c_{2}}\left(\begin{array}{c}
n \\
d
\end{array}\right) p_{1}^{d}\left(1-p_{1}\right)^{n-d}\right)\right\}
\end{aligned}
$$




$$
\begin{aligned}
P_{a}\left(p_{2}\right)= & \sum_{d=0}^{c_{1}}\left(\begin{array}{l}
n \\
d
\end{array}\right) p_{2}^{d}\left(1-p_{2}\right)^{n-d}+\sum_{d=c_{1}+1}^{c_{2}}\left(\begin{array}{l}
n \\
d
\end{array}\right) p_{2}^{d}\left(1-p_{2}\right)^{n-d} \\
& \cdot\left\{\left(\sum_{d=0}^{c_{1}}\left(\begin{array}{l}
n \\
d
\end{array}\right) p_{2}^{d}\left(1-p_{2}\right)^{n-d}\right)^{m}+m\left(\sum_{d=0}^{c_{1}}\left(\begin{array}{l}
n \\
d
\end{array}\right) p_{2}^{d}\left(1-p_{2}\right)^{n-d}\right)^{m-1}\left(\sum_{d=c_{1}+1}^{c_{2}}\left(\begin{array}{l}
n \\
d
\end{array}\right) p_{2}^{d}\left(1-p_{2}\right)^{n-d}\right)\right\} .
\end{aligned}
$$

Under binomial distribution, the proposed plan is designed for specified values of $p_{1}$ and $p_{2}$. Under Weibull and Birnbaum-Saunders distributions, the values of $p_{1}$ and $p_{2}$ are calculated using equations (4)-(8), respectively, for different ratios of a true mean lifetime and the specified lifetime of the product $\mu / \mu_{0}$.

We determine the values of $n, c_{1}, c_{2}$, and $m$ of the proposed modified MDS sampling plan under three distributions, namely, binomial, Weibull, and BS distributions. The optimal parameters are selected so that the risks of both producer and consumer are satisfied simultaneously with the minimum sample size. Also, the optimal parameters are determined for some randomly selected values of the producer's risk, consumer's risk, shape parameter of the distributions, and experiment termination ratio. In this design, the producer risk is fixed as $\alpha=0.05$ with different values of consumer risks. That is, under binomial distribution, the consumer's risk is taken as the only $\beta=0.10$ but, in the other two distributions, four values of $\beta$ are considered, $0.25,0.10,0.05$, and 0.01 , and also two cases of experiment termination ratio are considered as $a=0.5$ and $a=1.0$. Under BS distribution, the values of shape parameter $\omega=1.0$ and $\omega=1.5$ are chosen and the values of shape parameter for Weibull distribution are $\delta=1.2,1.5$, and 2.0. Optimal parameters of the proposed plan obtained under binomial distribution for specified values of AQL and LQL are provided in Table 1. From this table, we can observe that, for the same AQL value, the sample size decreases when there is an increment in LQL. Tables 2-6 report the optimal parameters of the proposed plan obtained under BS distribution and Weibull distribution, respectively.

3.2.1. Observations from Tables 2 and 3. For fixed values of $a$ and $\mu / \mu_{0}$, the sample size increases if there is either a decrement in consumer's risk or an increment in the shape parameter value. There is no certain trend in sample size when $a$ increases. The sample size is reduced if the mean ratio increases.

3.2.2. Observations from Tables 4-6. For fixed values of $\beta$ and $\mu / \mu_{0}$, the sample size decreases if either experiment termination ratio or mean ratio increases. When $\beta$ decreases, the sample size is increased. The sample size decreases or remains constant if the shape parameter changes from 1.2 to 1.5. From all tables, no certain trend can be observed in the value of $m$.

3.3. Example. In recent days, statistical distributions are frequently used in modeling fatigue data or failure time data because the lifetimes of a fatiguing material can vary due to manufacturing variation or random flaws in the material. In addition, BS distribution is considered as a suitable model for fatigue data as well as failure time data representation among other distributions and also this distribution is referred to as the fatigue-life distribution (see [18]). Therefore, the quality inspector wants to implement the proposed modified MDS sampling plan to inspect the breakdown times (in minutes) of electrical insulating fluid at $38 \mathrm{kV}$ under BS distribution based on time truncated life test. The target average breakdown time of electrical insulating fluids is specified as $\mu_{0}=40$ (i.e., $\mu_{0}=40$ ) minutes. It is assumed that the shape parameter of the distribution of breakdown time of electrical insulating fluids is $\omega=1.0$. Suppose that the consumer will accept a lot of products with probability 0.05 when the true breakdown time is equal to $\mu_{0}$. The producer would like the lot of products to be accepted with a probability higher than 0.95 when $\mu=160$ minutes. Suppose that the quality personnel prefer to utilize the proposed modified MDS sampling plan to guarantee that the average breakdown time of electrical insulating fluids is at least $\mu_{0}$ by using a truncated life test during 20 minutes. The aforementioned information leads to $\alpha=0.05, \beta=0.05$, $\mu / \mu_{0}=4$, and $a=0.5$. From Table 2, it can be selected that modified MDS sampling plan parameters are $n=7, c_{1}=0$, $c_{2}=1$, and $m=2$. For implementation purpose, we consider a real data set given by [24]. The breakdown time of 8 electrical insulating fluids is provided but for illustration purpose, the breakdown time of 7 electrical insulating fluids is considered, and we slightly modify the breakdown times of electrical insulating fluids. It is found that the breakdown time of 7 electrical insulating fluids is well fitted to a BS distribution with shape parameter $1.00054 \approx 1.0$, that is, $\widehat{\omega}=1.0$. The implementation of the proposed plan for testing the breakdown time of electrical insulating fluids is as follows.

A random sample of 7 electrical insulating fluids is selected from the current lot and performs the life-testing operations for 20 minutes. Observe and record the breakdown time of electrical insulating fluids. Suppose that the breakdown time of electrical insulating fluids at $38 \mathrm{kV}$ is given as follows:

\begin{tabular}{lllllll}
\hline 0.29 & 0.39 & 0.47 & 1.28 & 2.38 & 2.73 & 3.5 \\
\hline
\end{tabular}

It is observed from the above data that the breakdown time of none of the sampled electrical insulating fluids is less than 20 minutes. Therefore, we accept the current lot, since $d=0\left(=c_{1}\right)$.

\section{Comparative Study}

4.1. Comparison Using ASN. In order to show the better performance of the proposed modified MDS sampling plan in reducing the ASN compared to existing plans such as MDS sampling plan and SSP, the ASN of these plans are reported in 
TABLE 1: Plan parameters of modified MDS sampling plan for binomial distribution with $\beta=0.10$.

\begin{tabular}{|c|c|c|c|c|c|c|c|}
\hline$p_{1}$ & $p_{2}$ & $n$ & $c_{1}$ & $c_{2}$ & $m$ & $P_{a}\left(p_{1}\right)$ & $P_{a}\left(p_{2}\right)$ \\
\hline \multirow{3}{*}{0.0025} & 0.025 & 92 & 0 & 2 & 4 & 0.9590 & 0.0983 \\
\hline & 0.030 & 76 & 0 & 1 & 4 & 0.9564 & 0.0990 \\
\hline & 0.050 & 45 & 0 & 1 & 4 & 0.9867 & 0.0997 \\
\hline \multirow{3}{*}{0.005} & 0.04 & 59 & 0 & 2 & 3 & 0.9541 & 0.0961 \\
\hline & 0.05 & 46 & 0 & 2 & 4 & 0.9589 & 0.0953 \\
\hline & 0.10 & 22 & 0 & 1 & 4 & 0.9874 & 0.0987 \\
\hline \multirow{4}{*}{0.010} & 0.05 & 77 & 1 & 3 & 4 & 0.9635 & 0.0978 \\
\hline & 0.10 & 22 & 0 & 2 & 4 & 0.9630 & 0.0996 \\
\hline & 0.15 & 15 & 0 & 1 & 2 & 0.9857 & 0.0985 \\
\hline & 0.20 & 11 & 0 & 1 & 2 & 0.9928 & 0.0972 \\
\hline \multirow{4}{*}{0.03} & 0.09 & 60 & 2 & 7 & 3 & 0.9518 & 0.0970 \\
\hline & 0.12 & 32 & 1 & 4 & 3 & 0.9581 & 0.0981 \\
\hline & 0.15 & 25 & 1 & 3 & 3 & 0.9787 & 0.0971 \\
\hline & 0.30 & 7 & 0 & 1 & 2 & 0.9716 & 0.0941 \\
\hline \multirow{4}{*}{0.05} & 0.15 & 35 & 2 & 6 & 3 & 0.9571 & 0.0970 \\
\hline & 0.20 & 19 & 1 & 4 & 3 & 0.9604 & 0.0904 \\
\hline & 0.25 & 14 & 0 & 2 & 1 & 0.9555 & 0.0918 \\
\hline & 0.50 & 4 & 0 & 1 & 2 & 0.9762 & 0.0713 \\
\hline
\end{tabular}

TABle 2: Plan parameters of modified MDS sampling plan for Birnbaum-Saunders distribution with $\omega=1.0$.

\begin{tabular}{|c|c|c|c|c|c|c|c|c|c|c|c|c|c|}
\hline \multirow{2}{*}{$\beta$} & \multirow{2}{*}{$\mu / \mu_{0}$} & \multicolumn{5}{|c|}{$a=0.5$} & \multicolumn{7}{|c|}{$a=1.0$} \\
\hline & & $n$ & $c_{1}$ & $c_{2}$ & $M$ & $P_{a}\left(p_{1}\right)$ & $P_{a}\left(p_{2}\right)$ & $n$ & $c_{1}$ & $c_{2}$ & $m$ & $P_{a}\left(p_{1}\right)$ & $P_{a}\left(p_{2}\right)$ \\
\hline \multirow{5}{*}{0.25} & 2 & 11 & 2 & 4 & 2 & 0.9650 & 0.1978 & 9 & 4 & 6 & 2 & 0.9604 & 0.2441 \\
\hline & 4 & 3 & 0 & 1 & 4 & 0.9931 & 0.2416 & 4 & 0 & 2 & 1 & 0.9811 & 0.1860 \\
\hline & 6 & $\uparrow$ & $\uparrow$ & $\uparrow$ & $\uparrow$ & 0.9998 & $\uparrow$ & 2 & 0 & 1 & 2 & 0.9925 & 0.1700 \\
\hline & 8 & $\uparrow$ & $\uparrow$ & $\uparrow$ & $\uparrow$ & 1.0000 & $\uparrow$ & $\uparrow$ & $\uparrow$ & $\uparrow$ & $\uparrow$ & 0.9988 & $\uparrow$ \\
\hline & 10 & $\uparrow$ & $\uparrow$ & $\uparrow$ & $\uparrow$ & 1.0000 & $\uparrow$ & $\uparrow$ & $\uparrow$ & $\uparrow$ & $\uparrow$ & 0.9998 & $\uparrow$ \\
\hline \multirow{5}{*}{0.10} & 2 & 16 & 3 & 5 & 2 & 0.9558 & 0.0955 & 15 & 6 & 11 & 2 & 0.9558 & 0.0803 \\
\hline & 4 & 5 & 0 & 1 & 3 & 0.9814 & 0.0889 & 5 & 0 & 2 & 1 & 0.9562 & 0.0530 \\
\hline & 6 & $\uparrow$ & $\uparrow$ & $\uparrow$ & $\uparrow$ & 0.9994 & $\uparrow$ & 3 & 0 & 1 & 2 & 0.9775 & 0.0444 \\
\hline & 8 & $\uparrow$ & $\uparrow$ & $\uparrow$ & $\uparrow$ & 1.0000 & $\uparrow$ & $\uparrow$ & $\uparrow$ & $\uparrow$ & $\uparrow$ & 0.9962 & $\uparrow$ \\
\hline & 10 & $\uparrow$ & $\uparrow$ & $\uparrow$ & $\uparrow$ & 1.0000 & $\uparrow$ & $\uparrow$ & $\uparrow$ & $\uparrow$ & $\uparrow$ & 0.9993 & $\uparrow$ \\
\hline \multirow{5}{*}{0.05} & 2 & 19 & 3 & 7 & 2 & 0.9568 & 0.0460 & 19 & 8 & 12 & 2 & 0.9650 & 0.0415 \\
\hline & 4 & 7 & 0 & 1 & 2 & 0.9710 & 0.0343 & 7 & 0 & 3 & 1 & 0.9779 & 0.0351 \\
\hline & 6 & $\uparrow$ & $\uparrow$ & $\uparrow$ & $\uparrow$ & 0.9989 & $\uparrow$ & 3 & 0 & 1 & 2 & 0.9775 & 0.0444 \\
\hline & 8 & $\uparrow$ & $\uparrow$ & $\uparrow$ & $\uparrow$ & 0.9999 & $\uparrow$ & $\uparrow$ & $\uparrow$ & $\uparrow$ & $\uparrow$ & 0.9962 & $\uparrow$ \\
\hline & 10 & $\uparrow$ & $\uparrow$ & $\uparrow$ & $\uparrow$ & 1.0000 & $\uparrow$ & $\uparrow$ & $\uparrow$ & $\uparrow$ & $\uparrow$ & 0.9993 & $\uparrow$ \\
\hline \multirow{5}{*}{0.01} & 2 & 30 & 5 & 9 & 2 & 0.9552 & 0.0093 & 27 & 11 & 16 & 2 & 0.9513 & 0.0077 \\
\hline & 4 & 10 & 0 & 2 & 2 & 0.9777 & 0.0081 & 8 & 0 & 3 & 1 & 0.9602 & 0.0095 \\
\hline & 6 & 10 & 0 & 1 & 2 & 0.9976 & 0.0076 & 5 & 0 & 1 & 1 & 0.9512 & 0.0069 \\
\hline & 8 & $\uparrow$ & $\uparrow$ & $\uparrow$ & $\uparrow$ & 0.9999 & $\uparrow$ & $\uparrow$ & $\uparrow$ & $\uparrow$ & $\uparrow$ & 0.9902 & $\uparrow$ \\
\hline & 10 & $\uparrow$ & $\uparrow$ & $\uparrow$ & $\uparrow$ & 1.0000 & $\uparrow$ & $\uparrow$ & $\uparrow$ & $\uparrow$ & $\uparrow$ & 0.9979 & $\uparrow$ \\
\hline
\end{tabular}

$\uparrow$ : use the plan above.

Table 7. ASN of the MDS sampling plan is obtained from [16]. Also, the ASN of SSP is calculated by substituting $c_{2}=c_{1}=c$ (say) in OC function of modified MDS sampling plan. The following trends can be observed in Table 7:

(i) ASN of the proposed plan is smaller than the ASN of the MDS sampling plan when $\mu / \mu_{0}=2$. Also, for most of the AQL and LQL combinations, the ASN of the proposed plan is small when compared with the ASN of SSP. For example, when $\alpha=0.05, \beta=0.01, \delta=2, a=0.5$, and $\mu / \mu_{0}=4$, the ASN of the proposed plan is 24 . The respective ASN of the MDS sampling plan and SSP are 35 and 44.

(ii) ASN of all those plans coincide when the mean ratio increases. This represents that all three plans require the same sample size when the product's quality is good. However, the producer's risk is greatly reduced under the proposed sampling plan when the quality level is good. 
TABLE 3: Plan parameters of modified MDS sampling plan for Birnbaum-Saunders distribution with $\omega=1.5$.

\begin{tabular}{|c|c|c|c|c|c|c|c|c|c|c|c|c|c|}
\hline \multirow{2}{*}{$\beta$} & \multirow{2}{*}{$\mu / \mu_{0}$} & \multicolumn{5}{|c|}{$a=0.5$} & \multicolumn{7}{|c|}{$a=1.0$} \\
\hline & & $n$ & $c_{1}$ & $c_{2}$ & $M$ & $P_{a}\left(p_{1}\right)$ & $P_{a}\left(p_{2}\right)$ & $n$ & $c_{1}$ & $c_{2}$ & $m$ & $P_{a}\left(p_{1}\right)$ & $P_{a}\left(p_{2}\right)$ \\
\hline \multirow{5}{*}{0.25} & 2 & 18 & 7 & 10 & 3 & 0.9525 & 0.2352 & 20 & 11 & 15 & 2 & 0.9614 & 0.2320 \\
\hline & 4 & 7 & 0 & 3 & 1 & 0.9669 & 0.2193 & 8 & 0 & 5 & 1 & 0.9614 & 0.2089 \\
\hline & 6 & 2 & 0 & 1 & 4 & 0.9602 & 0.2484 & 5 & 0 & 3 & 1 & 0.9787 & 0.2307 \\
\hline & 8 & $\uparrow$ & $\uparrow$ & $\uparrow$ & $\uparrow$ & 0.9893 & $\uparrow$ & 4 & 0 & 2 & 1 & 0.9736 & 0.1311 \\
\hline & 10 & $\uparrow$ & $\uparrow$ & $\uparrow$ & $\uparrow$ & 0.9970 & $\uparrow$ & 2 & 0 & 1 & 2 & 0.9656 & 0.1286 \\
\hline \multirow{5}{*}{0.10} & 2 & 31 & 11 & 16 & 2 & 0.9505 & 0.0821 & 30 & 17 & 21 & 3 & 0.9525 & 0.0978 \\
\hline & 4 & 11 & 2 & 5 & 2 & 0.9693 & 0.0354 & 12 & 0 & 7 & 1 & 0.9625 & 0.0815 \\
\hline & 6 & 4 & 0 & 2 & 2 & 0.9588 & 0.0972 & 8 & 0 & 4 & 1 & 0.9612 & 0.0402 \\
\hline & 8 & 4 & 0 & 1 & 2 & 0.9690 & 0.0615 & 6 & 0 & 3 & 1 & 0.9840 & 0.0693 \\
\hline & 10 & $\uparrow$ & $\uparrow$ & $\uparrow$ & $\uparrow$ & 0.9901 & $\uparrow$ & 3 & 0 & 2 & 2 & 0.9595 & 0.0509 \\
\hline \multirow{5}{*}{0.05} & 2 & 39 & 14 & 19 & 2 & 0.9511 & 0.0462 & 38 & 21 & 29 & 3 & 0.9506 & 0.0467 \\
\hline & 4 & 11 & 2 & 5 & 2 & 0.9693 & 0.0354 & 14 & 5 & 9 & 2 & 0.9641 & 0.0122 \\
\hline & 6 & 7 & 0 & 2 & 1 & 0.9657 & 0.0454 & 8 & 0 & 4 & 1 & 0.9612 & 0.0402 \\
\hline & 8 & 5 & 0 & 2 & 2 & 0.9819 & 0.0373 & 7 & 0 & 3 & 1 & 0.9669 & 0.0173 \\
\hline & 10 & 5 & 0 & 1 & 2 & 0.9830 & 0.0277 & 5 & 0 & 2 & 1 & 0.9750 & 0.0303 \\
\hline \multirow{5}{*}{0.01} & 2 & 60 & 21 & 29 & 2 & 0.9529 & 0.0087 & $*$ & $*$ & $*$ & * & $*$ & $*$ \\
\hline & 4 & 16 & 3 & 6 & 2 & 0.9610 & 0.0080 & 16 & 6 & 9 & 2 & 0.9605 & 0.0083 \\
\hline & 6 & 10 & 1 & 3 & 2 & 0.9701 & 0.0085 & 11 & 0 & 5 & 1 & 0.9511 & 0.0068 \\
\hline & 8 & 7 & 0 & 2 & 2 & 0.9559 & 0.0067 & 10 & 0 & 4 & 1 & 0.9684 & 0.0025 \\
\hline & 10 & 7 & 0 & 1 & 1 & 0.9745 & 0.0086 & 6 & 0 & 2 & 1 & 0.9532 & 0.0061 \\
\hline
\end{tabular}

*plan does not exist. $\uparrow$ : use the plan above.

TABLE 4: Plan parameters of modified MDS sampling plan for Weibull distribution with $\delta=1.2$.

\begin{tabular}{|c|c|c|c|c|c|c|c|c|c|c|c|c|c|}
\hline \multirow{2}{*}{$\beta$} & \multirow{2}{*}{$\mu / \mu_{0}$} & \multicolumn{5}{|c|}{$a=0.5$} & \multicolumn{7}{|c|}{$a=1.0$} \\
\hline & & $n$ & $c_{1}$ & $c_{2}$ & $m$ & $P_{a}\left(p_{1}\right)$ & $P_{a}\left(p_{2}\right)$ & $n$ & $c_{1}$ & $c_{2}$ & $m$ & $P_{a}\left(p_{1}\right)$ & $P_{a}\left(p_{2}\right)$ \\
\hline \multirow{5}{*}{0.25} & 2 & 15 & 3 & 6 & 4 & 0.9500 & 0.2249 & 8 & 3 & 6 & 3 & 0.9545 & 0.2122 \\
\hline & 4 & 8 & 0 & 2 & 1 & 0.9756 & 0.2416 & 4 & 1 & 2 & 2 & 0.9807 & 0.2243 \\
\hline & 6 & 4 & 0 & 1 & 3 & 0.9716 & 0.2199 & 2 & 0 & 1 & 2 & 0.9794 & 0.2388 \\
\hline & 8 & $\uparrow$ & $\uparrow$ & $\uparrow$ & $\uparrow$ & 0.9870 & $\uparrow$ & $\uparrow$ & $\uparrow$ & $\uparrow$ & $\uparrow$ & 0.9905 & $\uparrow$ \\
\hline & 10 & $\uparrow$ & $\uparrow$ & $\uparrow$ & $\uparrow$ & 0.9930 & $\uparrow$ & $\uparrow$ & $\uparrow$ & $\uparrow$ & $\uparrow$ & 0.9948 & $\uparrow$ \\
\hline \multirow{5}{*}{0.10} & 2 & 26 & 5 & 9 & 3 & 0.9610 & 0.0993 & 13 & 5 & 8 & 3 & 0.9544 & 0.0988 \\
\hline & 4 & 11 & 1 & 3 & 3 & 0.9732 & 0.0787 & 5 & 1 & 2 & 2 & 0.9540 & 0.0932 \\
\hline & 6 & 6 & 0 & 2 & 3 & 0.9604 & 0.0970 & 4 & 0 & 2 & 2 & 0.9513 & 0.0364 \\
\hline & 8 & 6 & 0 & 1 & 3 & 0.9658 & 0.0901 & 3 & 0 & 1 & 2 & 0.9718 & 0.0725 \\
\hline & 10 & $\uparrow$ & $\uparrow$ & $\uparrow$ & $\uparrow$ & 0.9812 & $\uparrow$ & $\uparrow$ & $\uparrow$ & $\uparrow$ & $\uparrow$ & 0.9843 & $\uparrow$ \\
\hline \multirow{5}{*}{0.05} & 2 & 34 & 6 & 10 & 2 & 0.9523 & 0.0445 & 17 & 6 & 10 & 2 & 0.9556 & 0.0480 \\
\hline & 4 & 13 & 1 & 3 & 2 & 0.9687 & 0.0457 & 6 & 1 & 3 & 3 & 0.9510 & 0.0394 \\
\hline & 6 & 9 & 0 & 3 & 2 & 0.9581 & 0.0472 & 4 & 0 & 2 & 2 & 0.9513 & 0.0364 \\
\hline & 8 & 8 & 0 & 1 & 2 & 0.9538 & 0.0415 & $\uparrow$ & $\uparrow$ & $\uparrow$ & $\uparrow$ & 0.9796 & $\uparrow$ \\
\hline & 10 & $\uparrow$ & $\uparrow$ & $\uparrow$ & $\uparrow$ & 0.9738 & $\uparrow$ & 4 & 0 & 1 & 2 & 0.9682 & 0.0255 \\
\hline \multirow{5}{*}{0.01} & 2 & 52 & 9 & 15 & 2 & 0.9570 & 0.0098 & 26 & 9 & 15 & 2 & 0.9517 & 0.0095 \\
\hline & 4 & 22 & 0 & 4 & 1 & 0.9643 & 0.0096 & 11 & 0 & 4 & 1 & 0.9605 & 0.0087 \\
\hline & 6 & 15 & 0 & 2 & 1 & 0.9568 & 0.0086 & 7 & 0 & 2 & 1 & 0.9586 & 0.0097 \\
\hline & 8 & 12 & 0 & 2 & 2 & 0.9559 & 0.0083 & $\uparrow$ & $\uparrow$ & $\uparrow$ & $\uparrow$ & 0.9843 & $\uparrow$ \\
\hline & 10 & $\uparrow$ & $\uparrow$ & $\uparrow$ & $\uparrow$ & 0.9777 & $\uparrow$ & 5 & 0 & 2 & 3 & 0.9570 & 0.0096 \\
\hline
\end{tabular}

$\uparrow:$ use the plan above.

From this comparison, we conclude that the proposed plan will be very effective in protecting the producer when the product has good quality.

4.2. Comparison Using the OC Curve. In order to investigate the effect of the parameter $m$ on the probability of acceptance of the proposed plan, we consider the OC curves of the proposed plan having the same values of $n, c_{1}$, and $c_{2}$ but with different values of $m$. To draw the OC curves, we consider the optimal values of $n, c_{1}, c_{2}$, and $m$ such that the respective producer and consumer risks of 0.05 and 0.10 are satisfied at AQL (or $\left.p_{1}\right)=0.005$ and LQL $\left(\right.$ or $\left.p_{2}\right)=0.05$. The corresponding plan for the aforementioned specifications is $n=46, c_{1}=0, c_{2}=2$, and $m=4$ and OC curves for this plan 
TABLE 5: Plan parameters of modified MDS sampling plan for Weibull distribution with $\delta=1.5$.

\begin{tabular}{|c|c|c|c|c|c|c|c|c|c|c|c|c|c|}
\hline \multirow{2}{*}{$\beta$} & \multirow{2}{*}{$\mu / \mu_{0}$} & \multicolumn{5}{|c|}{$a=0.5$} & \multicolumn{7}{|c|}{$a=1.0$} \\
\hline & & $n$ & $c_{1}$ & $c_{2}$ & $m$ & $P_{a}\left(p_{1}\right)$ & $P_{a}\left(p_{2}\right)$ & $n$ & $c_{1}$ & $c_{2}$ & $m$ & $P_{a}\left(p_{1}\right)$ & $P_{a}\left(p_{2}\right)$ \\
\hline \multirow{5}{*}{0.25} & 2 & 15 & 2 & 4 & 3 & 0.9648 & 0.2350 & 6 & 2 & 4 & 4 & 0.9623 & 0.2290 \\
\hline & 4 & 5 & 0 & 1 & 3 & 0.9703 & 0.2455 & 2 & 0 & 1 & 3 & 0.9682 & 0.2059 \\
\hline & 6 & $\uparrow$ & $\uparrow$ & $\uparrow$ & $\uparrow$ & 0.9925 & $\uparrow$ & $\uparrow$ & $\uparrow$ & $\uparrow$ & $\uparrow$ & 0.9924 & $\uparrow$ \\
\hline & 8 & $\uparrow$ & $\uparrow$ & $\uparrow$ & $\uparrow$ & 0.9972 & $\uparrow$ & $\uparrow$ & $\uparrow$ & $\uparrow$ & $\uparrow$ & 0.9973 & $\uparrow$ \\
\hline & 10 & $\uparrow$ & $\uparrow$ & $\uparrow$ & $\uparrow$ & 0.9987 & $\uparrow$ & $\uparrow$ & $\uparrow$ & $\uparrow$ & $\uparrow$ & 0.9988 & $\uparrow$ \\
\hline \multirow{5}{*}{0.10} & 2 & 24 & 3 & 6 & 3 & 0.9614 & 0.0985 & 10 & 3 & 5 & 2 & 0.9526 & 0.0957 \\
\hline & 4 & 8 & 0 & 2 & 3 & 0.9525 & 0.0962 & 4 & 0 & 2 & 2 & 0.9524 & 0.0513 \\
\hline & 6 & 8 & 0 & 1 & 3 & 0.9771 & 0.0900 & 3 & 0 & 1 & 2 & 0.9845 & 0.0928 \\
\hline & 8 & $\uparrow$ & $\uparrow$ & $\uparrow$ & $\uparrow$ & 0.9914 & $\uparrow$ & $\uparrow$ & $\uparrow$ & $\uparrow$ & $\uparrow$ & 0.9940 & $\uparrow$ \\
\hline & 10 & $\uparrow$ & $\uparrow$ & $\uparrow$ & $\uparrow$ & 0.9960 & $\uparrow$ & $\uparrow$ & $\uparrow$ & $\uparrow$ & $\uparrow$ & 0.9971 & $\uparrow$ \\
\hline \multirow{5}{*}{0.05} & 2 & 29 & 3 & 7 & 2 & 0.9514 & 0.0493 & 13 & 4 & 7 & 3 & 0.9572 & 0.0490 \\
\hline & 4 & 11 & 0 & 2 & 2 & 0.9514 & 0.0466 & 7 & 0 & 2 & 1 & 0.9597 & 0.0169 \\
\hline & 6 & 10 & 0 & 1 & 3 & 0.9620 & 0.0484 & 4 & 0 & 1 & 2 & 0.9685 & 0.0345 \\
\hline & 8 & $\uparrow$ & $\uparrow$ & $\uparrow$ & $\uparrow$ & 0.9855 & $\uparrow$ & $\uparrow$ & $\uparrow$ & $\uparrow$ & $\uparrow$ & 0.9877 & $\uparrow$ \\
\hline & 10 & $\uparrow$ & $\uparrow$ & $\uparrow$ & $\uparrow$ & 0.9932 & $\uparrow$ & $\uparrow$ & $\uparrow$ & $\uparrow$ & $\uparrow$ & 0.9941 & $\uparrow$ \\
\hline \multirow{5}{*}{0.01} & 2 & 47 & 5 & 10 & 2 & 0.9510 & 0.0091 & 21 & 6 & 10 & 2 & 0.9612 & 0.0076 \\
\hline & 4 & 23 & 1 & 3 & 2 & 0.9785 & 0.0087 & 9 & 1 & 3 & 2 & 0.9763 & 0.0061 \\
\hline & 6 & 16 & 0 & 2 & 2 & 0.9726 & 0.0082 & 6 & 0 & 2 & 2 & 0.9700 & 0.0063 \\
\hline & 8 & 16 & 0 & 1 & 2 & 0.9694 & 0.0078 & 6 & 0 & 1 & 1 & 0.9778 & 0.0083 \\
\hline & 10 & $\uparrow$ & $\uparrow$ & $\uparrow$ & $\uparrow$ & 0.9851 & $\uparrow$ & $\uparrow$ & $\uparrow$ & $\uparrow$ & $\uparrow$ & 0.9886 & $\uparrow$ \\
\hline
\end{tabular}

$\uparrow:$ use the plan above.

TABLE 6: Plan parameters of modified MDS sampling plan for Weibull distribution with $\delta=2.0$.

\begin{tabular}{|c|c|c|c|c|c|c|c|c|c|c|c|c|c|}
\hline \multirow{2}{*}{$\beta$} & \multirow{2}{*}{$\mu / \mu_{0}$} & \multicolumn{5}{|c|}{$a=0.5$} & \multicolumn{7}{|c|}{$a=1.0$} \\
\hline & & $n$ & $c_{1}$ & $c_{2}$ & $m$ & $P_{a}\left(p_{1}\right)$ & $P_{a}\left(p_{2}\right)$ & $n$ & $c_{1}$ & $c_{2}$ & $m$ & $P_{a}\left(p_{1}\right)$ & $P_{a}\left(p_{2}\right)$ \\
\hline \multirow{5}{*}{0.25} & 2 & 15 & 1 & 2 & 3 & 0.9501 & 0.2368 & 5 & 1 & 3 & 3 & 0.9676 & 0.1602 \\
\hline & 4 & 8 & 0 & 1 & 3 & 0.9930 & 0.2280 & 2 & 0 & 1 & 3 & 0.9949 & 0.2442 \\
\hline & 6 & $\uparrow$ & $\uparrow$ & $\uparrow$ & $\uparrow$ & 0.9989 & $\uparrow$ & $\uparrow$ & $\uparrow$ & $\uparrow$ & $\uparrow$ & 0.9993 & $\uparrow$ \\
\hline & 8 & $\uparrow$ & $\uparrow$ & $\uparrow$ & $\uparrow$ & 0.9997 & $\uparrow$ & $\uparrow$ & $\uparrow$ & $\uparrow$ & $\uparrow$ & 0.9998 & $\uparrow$ \\
\hline & 10 & $\uparrow$ & $\uparrow$ & $\uparrow$ & $\uparrow$ & 0.9999 & $\uparrow$ & $\uparrow$ & $\uparrow$ & $\uparrow$ & $\uparrow$ & 0.9999 & $\uparrow$ \\
\hline \multirow{5}{*}{0.10} & 2 & 22 & 1 & 3 & 2 & 0.9520 & 0.0981 & 7 & 1 & 5 & 2 & 0.9520 & 0.0969 \\
\hline & 4 & 12 & 0 & 1 & 3 & 0.9818 & 0.0966 & 3 & 0 & 1 & 3 & 0.9847 & 0.0982 \\
\hline & 6 & $\uparrow$ & $\uparrow$ & $\uparrow$ & $\uparrow$ & 0.9971 & $\uparrow$ & $\uparrow$ & $\uparrow$ & $\uparrow$ & $\uparrow$ & 0.9977 & $\uparrow$ \\
\hline & 8 & $\uparrow$ & $\uparrow$ & $\uparrow$ & $\uparrow$ & 0.9992 & $\uparrow$ & $\uparrow$ & $\uparrow$ & $\uparrow$ & $\uparrow$ & 0.9994 & $\uparrow$ \\
\hline & 10 & $\uparrow$ & $\uparrow$ & $\uparrow$ & $\uparrow$ & 0.9997 & $\uparrow$ & $\uparrow$ & $\uparrow$ & $\uparrow$ & $\uparrow$ & 0.9998 & $\uparrow$ \\
\hline \multirow{5}{*}{0.05} & 2 & 34 & 2 & 4 & 2 & 0.9614 & 0.0480 & 10 & 2 & 4 & 2 & 0.9565 & 0.0337 \\
\hline & 4 & 16 & 0 & 1 & 2 & 0.9747 & 0.0454 & 4 & 0 & 1 & 2 & 0.9783 & 0.0473 \\
\hline & 6 & $\uparrow$ & $\uparrow$ & $\uparrow$ & $\uparrow$ & 0.9956 & $\uparrow$ & $\uparrow$ & $\uparrow$ & $\uparrow$ & $\uparrow$ & 0.9963 & $\uparrow$ \\
\hline & 8 & $\uparrow$ & $\uparrow$ & $\uparrow$ & $\uparrow$ & 0.9987 & $\uparrow$ & $\uparrow$ & $\uparrow$ & $\uparrow$ & $\uparrow$ & 0.9989 & $\uparrow$ \\
\hline & 10 & $\uparrow$ & $\uparrow$ & $\uparrow$ & $\uparrow$ & 0.9995 & $\uparrow$ & $\uparrow$ & $\uparrow$ & $\uparrow$ & $\uparrow$ & 0.9996 & $\uparrow$ \\
\hline \multirow{5}{*}{0.01} & 2 & 53 & 3 & 6 & 2 & 0.9680 & 0.0099 & 15 & 3 & 6 & 2 & 0.9673 & 0.0077 \\
\hline & 4 & 24 & 0 & 2 & 2 & 0.9794 & 0.0095 & 6 & 0 & 2 & 3 & 0.9562 & 0.0090 \\
\hline & 6 & 24 & 0 & 1 & 2 & 0.9892 & 0.0090 & 6 & 0 & 1 & 2 & 0.9903 & 0.0091 \\
\hline & 8 & $\uparrow$ & $\uparrow$ & $\uparrow$ & $\uparrow$ & 0.9969 & $\uparrow$ & $\uparrow$ & $\uparrow$ & $\uparrow$ & $\uparrow$ & 0.9972 & $\uparrow$ \\
\hline & 10 & $\uparrow$ & $\uparrow$ & $\uparrow$ & $\uparrow$ & 0.9989 & $\uparrow$ & $\uparrow$ & $\uparrow$ & $\uparrow$ & $\uparrow$ & 0.9989 & $\uparrow$ \\
\hline
\end{tabular}

$\uparrow:$ use the plan above.

with $m=1,2,3$, and 4 are shown in Figure 1. It is observed from this figure that the OC curves of the proposed plan with $m=2,3$, and 4 are dominated by the OC curve of the plan with $m=1$. This represents that the chance for accepting the current lot is high if it depends only on the preceding lot acceptance. When proportion nonconforming increases, the OC curve with $m=i$ moves toward the OC curve with $m=i+1$, where $i=2$ and 3 . This shows that the value of $m$ does not make a great impact on acceptance probability of the current lot if proportion nonconforming increases. It is to be mentioned that, for a large value of $m$, the OC curve will approach an ideal OC curve. It is concluded from this comparison that the impact of the parameter $m$ in the modified MDS sampling plan is significant.

Further, to compare the performance of the proposed plan in discriminating lots based on the quality with existing 
TABLE 7: ASN of the proposed modified MDS (MMDS) plan, MDS sampling plan, and SSP for assuring Weibull distributed mean life with shape parameters $\delta=2$ and $a=0.5$.

\begin{tabular}{|c|c|c|c|c|c|c|c|}
\hline \multirow{2}{*}{$\beta$} & \multirow{2}{*}{$\mu / \mu_{0}$} & \multicolumn{2}{|c|}{ MMDS } & \multicolumn{2}{|c|}{ MDS } & \multicolumn{2}{|c|}{ SSP } \\
\hline & & ASN & $P_{a}\left(p_{1}\right)$ & ASN & $P_{a}\left(p_{1}\right)$ & ASN & $P_{a}\left(p_{1}\right)$ \\
\hline \multirow{5}{*}{0.25} & 2 & 15 & 0.9501 & 17 & 0.9560 & 28 & 0.9570 \\
\hline & 4 & 8 & 0.9930 & 8 & 0.9801 & 15 & 0.9859 \\
\hline & 6 & $\uparrow$ & 0.9989 & $\uparrow$ & 0.9957 & 8 & 0.9573 \\
\hline & 8 & $\uparrow$ & 0.9997 & $\uparrow$ & 0.9986 & $\uparrow$ & 0.9758 \\
\hline & 10 & $\uparrow$ & 0.9999 & $\uparrow$ & 0.9994 & $\uparrow$ & 0.9844 \\
\hline \multirow{5}{*}{0.10} & 2 & 22 & 0.9520 & 29 & 0.9512 & 50 & 0.9684 \\
\hline & 4 & 12 & 0.9818 & 12 & 0.9583 & 21 & 0.9732 \\
\hline & 6 & $\uparrow$ & 0.9971 & $\uparrow$ & 0.9906 & $\uparrow$ & 0.9942 \\
\hline & 8 & $\uparrow$ & 0.9992 & $\uparrow$ & 0.9969 & 12 & 0.9639 \\
\hline & 10 & $\uparrow$ & 0.9997 & $\uparrow$ & 0.9987 & $\uparrow$ & 0.9767 \\
\hline \multirow{5}{*}{0.05} & 2 & 34 & 0.9614 & 42 & 0.9527 & 64 & 0.9669 \\
\hline & 4 & 16 & 0.9747 & 16 & 0.9551 & 25 & 0.9629 \\
\hline & 6 & $\uparrow$ & 0.9956 & $\uparrow$ & 0.9900 & $\uparrow$ & 0.9918 \\
\hline & 8 & $\uparrow$ & 0.9987 & $\uparrow$ & 0.9967 & 16 & 0.9521 \\
\hline & 10 & $\uparrow$ & 0.9995 & $\uparrow$ & 0.9986 & $\uparrow$ & 0.9691 \\
\hline \multirow{5}{*}{0.01} & 2 & 53 & 0.9680 & 62 & 0.9616 & 93 & 0.9656 \\
\hline & 4 & 24 & 0.9794 & 35 & 0.9871 & 44 & 0.9834 \\
\hline & 6 & 24 & 0.9892 & 24 & 0.9783 & 35 & 0.9844 \\
\hline & 8 & $\uparrow$ & 0.9969 & $\uparrow$ & 0.9927 & $\uparrow$ & 0.9948 \\
\hline & 10 & $\uparrow$ & 0.9989 & $\uparrow$ & 0.9969 & 24 & 0.9540 \\
\hline
\end{tabular}

$\uparrow$ : use the plan above.

sampling plans, the OC curve of the proposed plan along with the same of MDS sampling plan and SSP is portrayed in Figure 2. For this comparative study, the OC curves of aforementioned plans are drawn using same values of parameters $n=15, c_{1}=1, c_{2}=2$, and $m=3$ for modified MDS and MDS sampling plan and $n=15$ and $c=1$ for SSP. These parameters are obtained from Table 6 to ensure Weibull distributed mean life when the shape parameters $\delta=2$, $a=0.5, \alpha=0.05, \beta=0.25$, and $r_{1}=2$. It can be observed from Figure 2 that the proposed plan yields more probability of acceptance rather than MDS sampling plan and SSP for small values of failure probability. Then the OC curve of proposed plan coincides with the other two OC curves for large values of failure probability. It is concluded from this comparison that the discriminating power of the proposed plan is better when compared to MDS sampling plan and SSP.

\section{Designing of Modified MDS Sampling Plan with an Economic Perspective}

Economic designing of sampling plans plays a significant role in acceptance sampling because in statistical designing only the two statistical errors, namely, rejection of good quality lot and the acceptance of poor quality, are considered. It does not consider the costs involved in the sampling plan implementation but, in economical designing, all the costs related to the plan implementation such as inspection cost per item, internal failure cost of an item, and the cost of an outgoing failure item are considered. In addition, the changes (either increment or decrement) in these costs and the plan parameters affect the total cost (TC) of an inspection. Therefore, in this section, we discuss the economical designing of a modified MDS sampling plan for assuring Weibull distributed mean life of the products. A number of authors have investigated the economic designing of various sampling plans using distinct approaches. For example, [25] presented a method for economical designing of acceptance sampling plans by considering continuous loss functions and inspection error. Aslam et al. [15] proposed the economic designing of a group acceptancesampling plan to assure Weibull distributed lifetime of the products using a Bayesian approach. Fallahnezhad et al. [26] provided the designing of repetitive group acceptance sampling plan with economic aspect by considering give-away cost per unit of sold excess material and inspection error. Balamurali et al. [27] proposed an economic model to select the optimal quick switching sampling system that minimizes TC while satisfying both the producer's and consumer's risk requirements. Hence, we consider the economic designing of the proposed sampling plan in this section. Some of the performance measures of the proposed plan are used in this economic designing, which are $P_{a}(p)$, the probability of acceptance, and the average total inspection (ATI) (see [28]). ATI is defined as follows:

$$
\mathrm{ATI}=n+\left(1-P_{a}(p)\right)(N-n)
$$

where the acceptance probability of the lot under the proposed modified MDS plan is obtained from equation (12). 


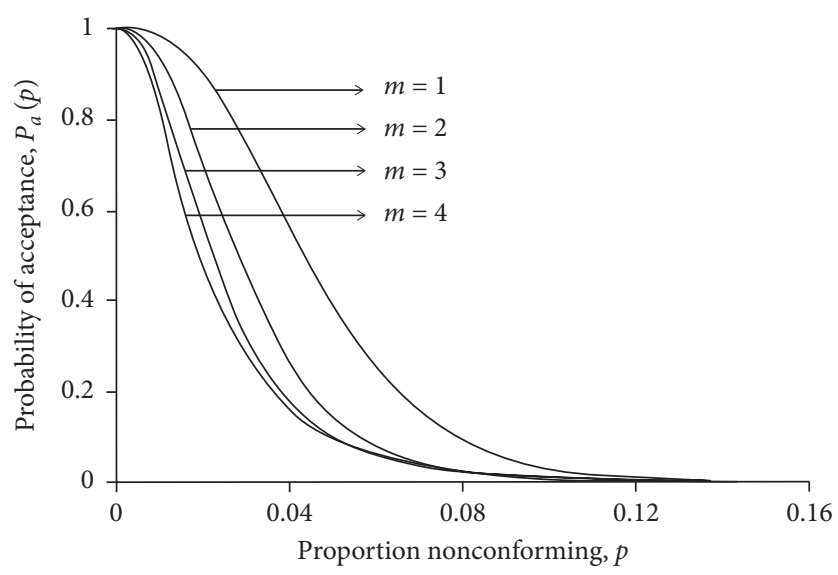

Figure 1: OC curves of the proposed plan with different $m$ values.

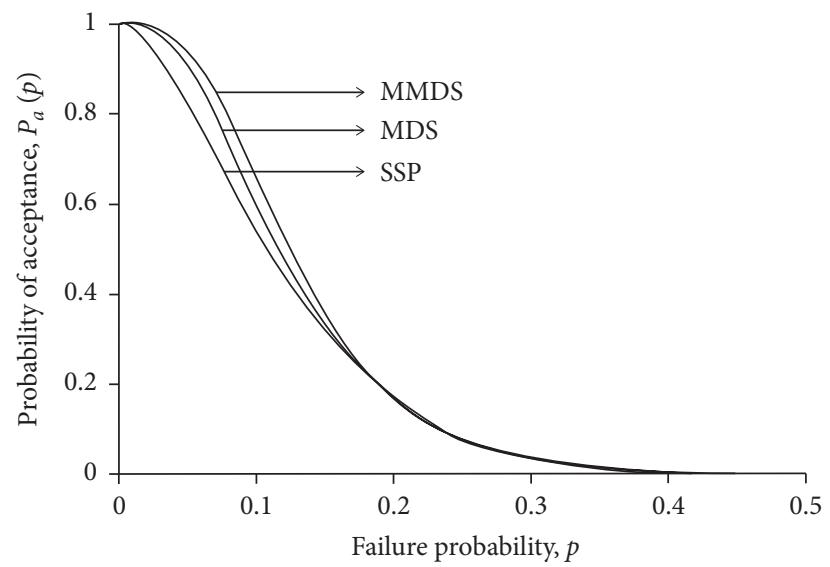

FIGURE 2: OC curves of the proposed plan, MDS sampling plan, and SSP.

Let us denote the detection and nondetection of failure items by $D_{d}$ and $D_{n}$, respectively. Then,

$$
\begin{aligned}
& D_{d}=n p+\left(1-P_{a}(p)\right)(N-n) p, \\
& D_{n}=p P_{a}(p)(N-n) .
\end{aligned}
$$

The costs involved in proposed plan implementation are defined as follows: $C_{i}=$ life testing cost per item; $C_{f}=$ cost of replacement; $C_{o}=$ cost of an outgoing failure item. It is important to note that the value of $p$ given in equations (16) and (17) is unknown in most of the cases. Under this situation, the sampling plans are designed for the specified value of $p$ or it is estimated using Bayesian approach. For example, [28] discussed the economic designing of SSP for specified values of $p$. Aslam et al. [15] investigated the group acceptance sampling plans with the economic aspect in which the value of $p$ has been estimated using the Bayesian approach. In this designing, we define $p$ as the failure probability corresponding to the average mean ratios of AQL and LQL.

The mathematical model for an economic modified MDS sampling plan is obtained as follows.

Minimize TC $=C_{i} \cdot \mathrm{ATI}+C_{f} \cdot D_{d}+C_{o} \cdot D_{n}$.

Subject to

$$
\begin{aligned}
& P_{a}\left(p_{1}\right) \geq 1-\alpha, \\
& P_{a}\left(p_{2}\right) \leq \beta, \\
& n>1, m \geq 1, c_{2}>c_{1} \geq 0,
\end{aligned}
$$

where we have $P_{a}\left(p_{1}\right)$ and $P_{a}\left(p_{2}\right)$ are the probabilities of acceptance of the lot at AQL and LQL given in equations (14) and (15), respectively.

5.1. Descriptive Example. Optimal parameters of the proposed modified MDS sampling plan such as $n, c_{1}, c_{2}$, and $m$ along with corresponding ATI, $D_{d}, D_{n}$, TC, and $P_{a}(p)$ are reported in Table 8 . The optimal parameters are determined for specified values of $a=0.5, \delta=2$, and lot size $N=1000$ and $C_{i}=1.0, C_{f}=2.0$, and $C_{o}=10$ as used by [28]. In general, the probabilities of failure corresponding to the mean ratios $\mu / \mu_{0}=2,4,6,8,10$ are considered as AQL and the probability of failure at the ratio $\mu / \mu_{0}=1$ is taken as LQL. The fixed value of the producer's risk is $\alpha=0.05$ and the consumer's risks are assumed to be $\beta=0.25,0.10,0.05,0.01$. As mentioned earlier, $p$ is defined as the failure probability corresponding to the average mean ratios of AQL and LQL. Therefore, the values of $p$ are the failure probabilities corresponding to the mean 
TABLE 8: Optimal parameters of economic modified MDS sampling plan for assuring Weibull distributed mean life with $a=0.5$ and $\delta=2$.

\begin{tabular}{|c|c|c|c|c|c|c|c|c|c|c|}
\hline$\beta$ & $\mu / \mu_{0}$ & $n$ & $c_{1}$ & $c_{2}$ & $m$ & ATI & $D_{d}$ & $D_{n}$ & TC & $P_{a}(p)$ \\
\hline \multirow{5}{*}{0.25} & 2 & 48 & 6 & 12 & 4 & 53.50 & 4.47 & 79.10 & 853.40 & 0.9942 \\
\hline & 4 & 15 & 1 & 4 & 4 & 17.50 & 0.54 & 30.39 & 322.45 & 0.9975 \\
\hline & 6 & 15 & 1 & 4 & 4 & 15.07 & 0.24 & 15.66 & 172.16 & 0.9999 \\
\hline & 8 & 8 & 0 & 4 & 4 & 10.24 & 0.09 & 9.55 & 105.94 & 0.9977 \\
\hline & 10 & 8 & 0 & 4 & 4 & 8.72 & 0.06 & 6.41 & 72.97 & 0.9993 \\
\hline \multirow{5}{*}{0.10} & 2 & 65 & 7 & 13 & 3 & 78.98 & 6.60 & 76.97 & 861.85 & 0.9851 \\
\hline & 4 & 21 & 1 & 4 & 3 & 28.26 & 0.87 & 30.05 & 330.54 & 0.9926 \\
\hline & 6 & 21 & 1 & 4 & 3 & 21.25 & 0.34 & 15.56 & 177.56 & 0.9997 \\
\hline & 8 & 14 & 0 & 2 & 2 & 16.38 & 0.16 & 9.49 & 111.61 & 0.9976 \\
\hline & 10 & 12 & 0 & 5 & 4 & 14.25 & 0.09 & 6.38 & 78.21 & 0.9977 \\
\hline \multirow{5}{*}{0.05} & 2 & 82 & 8 & 14 & 2 & 99.24 & 8.29 & 75.27 & 868.56 & 0.9812 \\
\hline & 4 & 34 & 2 & 10 & 3 & 35.80 & 1.11 & 29.82 & 336.22 & 0.9981 \\
\hline & 6 & 25 & 1 & 4 & 3 & 25.64 & 0.41 & 15.49 & 181.39 & 0.9993 \\
\hline & 8 & 17 & 0 & 2 & 2 & 21.12 & 0.20 & 9.45 & 115.98 & 0.9958 \\
\hline & 10 & 17 & 0 & 2 & 2 & 18.32 & 0.12 & 6.35 & 82.07 & 0.9987 \\
\hline \multirow{5}{*}{0.01} & 2 & 110 & 10 & 17 & 2 & 142.46 & 11.90 & 71.66 & 882.89 & 0.9635 \\
\hline & 4 & 45 & 2 & 6 & 2 & 49.59 & 1.53 & 29.39 & 346.60 & 0.9952 \\
\hline & 6 & 35 & 1 & 4 & 2 & 36.42 & 0.58 & 15.32 & 190.80 & 0.9985 \\
\hline & 8 & 30 & 0 & 2 & 1 & 33.64 & 0.32 & 9.32 & 127.54 & 0.9962 \\
\hline & 10 & 24 & 0 & 2 & 2 & 27.52 & 0.18 & 6.29 & 90.80 & 0.9964 \\
\hline
\end{tabular}

ratios $\mu / \mu_{0}=1.5,2.5,3.5,4.5$, and 5.5 . We can observe from the table that the values of $n$, ATI, $D_{d}, D_{n}$, and TC are indirectly proportional to both the mean ratio and the consumer's risk. That is, the values of $n, \mathrm{ATI}, D_{d}, D_{n}$, and TC are decreased if mean ratios increase but they are increased if there is a decrement in consumer's risk. There is no standard trend observed in probabilities of acceptance of the lot. That is, probabilities of acceptance are fluctuating when the mean ratio increases. In addition, the optimal parameters are selected so that both the producer and consumer risks are satisfied with minimum cost.

\section{Industrial Application}

Suppose that the ball bearing product manufacturer wants to adopt the proposed sampling plan to ensure the mean life of the manufactured products. The producer decides to conduct the fatigue-testing operations to know the fatigue life of the ball bearings, since the service life of ball bearings depends on the fatigue life (see [29]). The endurance life of ball bearings is measured in terms of million revolutions. The producer specified that the average number of million revolutions to fatigue of the ball bearing is $\mu_{0}=50$ million revolutions and it is decided to conduct the fatigue-testing operations for 25 million revolutions, that is, $t_{0}=25$. Therefore, the termination ratio of this test is calculated as $a=0.5$. The mean ratio is taken as $\mu / \mu_{0}=6$ and the producer and consumer risks are assumed to be $\alpha=0.05$ and $\beta=0.10$. For these above-specified values, Table 8 gives the optimal parameters of the economic modified MDS plan as follows: sample size $n=21$, unconditional acceptance number $c_{1}=1$, conditional acceptance number $c_{2}=4$, and number of preceding lots required for current lot disposition $m=3$. The operating procedure of the proposed plan is explained with the fatigue life data of ball bearings given by [29]. The following data represent the measurements of a number of million revolutions of 23 ball bearings before the fatigue. It is found that the data set is well fitted to a Weibull distribution, and the estimated value of shape parameter of these data is $2.088 \approx 2.0$; that is, $\widehat{\delta}=2$. For the illustration purpose, we consider 21 (i.e., from $3^{\text {rd }}$ to $23^{\text {rd }}$ ) measurements from data. The modified MDS sampling plan can be operated as follows.

Select a random sample of 21 ball bearings from the current lot and count the number of revolutions of ball bearings before fatigue. Suppose that the lifetime of 21 ball bearings is given as follows.

\begin{tabular}{lllllllllll}
33.00 & 41.52 & 42.12 & 45.60 & 48.48 & 51.84 & 51.96 & 54.12 & 55.56 & 67.80 & 60.64 \\
\hline
\end{tabular} \begin{tabular}{llllllllll}
\hline 68.64 & 68.88 & 84.12 & 93.12 & 98.64 & 105.12 & 105.84 & 127.92 & 128.04 & 173.40
\end{tabular}

From these data, it is observed that the fatigue life of all sampled ball bearings is greater than 25 million revolutions. Therefore, the current lot is immediately accepted without any condition, since $d=0<c_{1}$. The TC involved under the proposed sampling plan is 177.56 .

\section{Comparative Study Based on TC}

To show the efficiency of the proposed modified MDS sampling plan, the sample size and TC required for plan implementation are compared with those of MDS sampling plan proposed by [16] for assuring Weibull distributed mean life of the product and SSP. The values of sample size and TC are given in Table 9 and such values are determined for the specified values of shape parameters $\delta=2$ and $a=0.5$. From this table, it is observed that the sample sizes of the proposed plan and MDS sampling plan coincide when $\beta=0.25$ and the mean ratios are $\mu / \mu_{0}=6,10$ but the TC required under proposed plan is smaller than that of MDS sampling plan. For all the remaining combinations of $\beta$ and $\mu / \mu_{0}$, the sample size of the proposed plan is smaller than those of both the MDS sampling plan and SSP. A consequence of the minimum sample size, the TC of the proposed plan is minimum when compared with those of the other two plans. It is 
TABLE 9: Sample size and TC for proposed plan, MDS sampling plan, and SSP.

\begin{tabular}{|c|c|c|c|c|c|c|c|}
\hline \multirow{2}{*}{$\beta$} & \multirow{2}{*}{$\mu / \mu_{0}$} & \multicolumn{3}{|c|}{$n$} & \multicolumn{3}{|c|}{$\mathrm{TC}$} \\
\hline & & MMDS & MDS & SSP & MMDS & MDS & SSP \\
\hline \multirow{5}{*}{0.25} & 2 & 48 & 55 & 78 & 853.40 & 858.29 & 870.14 \\
\hline & 4 & 15 & 22 & 28 & 322.45 & 327.41 & 337.92 \\
\hline & 6 & $\uparrow$ & 15 & 21 & 172.16 & 173.07 & 181.02 \\
\hline & 8 & 8 & $\uparrow$ & $\uparrow$ & 105.94 & 110.49 & 116.82 \\
\hline & 10 & $\uparrow$ & 8 & 15 & 72.97 & 76.98 & 82.80 \\
\hline \multirow{5}{*}{0.10} & 2 & 65 & 78 & 110 & 861.85 & 869.11 & 884.13 \\
\hline & 4 & 21 & 29 & 43 & 330.54 & 336.12 & 349.02 \\
\hline & 6 & $\uparrow$ & 21 & 36 & 177.56 & 180.49 & 192.54 \\
\hline & 8 & 14 & $\uparrow$ & 29 & 111.61 & 116.41 & 125.69 \\
\hline & 10 & 12 & $\uparrow$ & 21 & 78.21 & 84.73 & 92.13 \\
\hline \multirow{5}{*}{0.05} & 2 & 82 & 99 & 132 & 868.56 & 876.98 & 893.57 \\
\hline & 4 & 34 & 42 & 57 & 336.22 & 343.18 & 358.02 \\
\hline & 6 & 25 & 27 & 42 & 181.39 & 186.58 & 199.36 \\
\hline & 8 & 17 & 25 & 34 & 115.98 & 120.60 & 131.70 \\
\hline & 10 & $\uparrow$ & $\uparrow$ & $\uparrow$ & 82.07 & 88.64 & 98.22 \\
\hline \multirow{5}{*}{0.01} & 2 & 110 & 110 & 193 & 882.89 & 902.97 & 914.13 \\
\hline & 4 & 45 & 54 & 78 & 346.60 & 356.01 & 375.30 \\
\hline & 6 & 35 & 44 & 53 & 190.80 & 199.18 & 213.60 \\
\hline & 8 & 30 & 35 & 44 & 127.54 & 130.89 & 144.91 \\
\hline & 10 & 24 & $\uparrow$ & $\uparrow$ & 90.80 & 98.38 & 109.09 \\
\hline
\end{tabular}

$\uparrow$ : use the plan above.

concluded from this comparison that the proposed plan is better than MDS sampling plan and SSP in terms of both sample size and TC.

\section{Conclusions}

In this paper, a modified version of the MDS sampling plan has been proposed and investigated to assure products' quality. In addition, the proposed modified MDS sampling plan is designed to ensure the mean life of the products under Weibull and Birnbaum-Saunders distributions. A nonlinear optimization problem has been used to determine the optimal parameters. The comparison between the proposed plan and existing sampling plans MDS and SSP shows that the proposed plan will reduce the producer's risk when product quality is good. The proposed plan with minimum ASN will also be more effective in reducing the cost of the inspection. So, it is recommended to use the proposed sampling plan for the inspection of products when the decision on the lot is based on the lifetime of the products.

\section{Data Availability}

The data are included within the article.

\section{Conflicts of Interest}

The authors declare that there are no conflicts of interest regarding the publication of this paper.

\section{Acknowledgments}

This work was funded by the Deanship of Scientific Research (DSR), King Abdulaziz University, Jeddah. The authors Muhammad Aslam and Ali AL-Marshadi, therefore, acknowledge with thanks the technical support of DSR. The author P. Jeyadurga would like to acknowledge the Kalasalingam Academy of Research and Education for providing financial support through postdoctoral fellowship.

\section{References}

[1] C.-H. Jun, S. Balamurali, and S.-H. Lee, "Variables sampling plans for Weibull distributed lifetimes under sudden death testing," IEEE Transactions on Reliability, vol. 55, no. 1, pp. 53-58, 2006.

[2] G. S. Rao, "A group acceptance sampling plans for lifetimes following a generalized exponential distribution," Economic Quality Control, vol. 24, no. 1, pp. 75-85, 2009.

[3] Y. L. Lio, T.-R. Tsai, and S.-J. Wu, "Acceptance sampling plans from truncated life tests based on the Birnbaum-Saunders distribution for percentiles," Communications in Statistics Simulation and Computation, vol. 39, no. 1, pp. 119-136, 2009.

[4] H. Rasay, M. Pourgharibshahi, and M. Fallahnezhad, "Sequential sampling plan in the truncated life test for Weibull distribution," Journal of Testing and Evaluation, vol. 46, no. 2, pp. 693-703, 2018.

[5] A. W. Wortham and R. C. Baker, "Multiple deferred state sampling inspection," International Journal of Production Research, vol. 14, no. 6, pp. 719-731, 1976.

[6] S. Balamurali and C.-H. Jun, "Multiple dependent state sampling plans for lot acceptance based on measurement data," European Journal of Operational Research, vol. 180, no. 3, pp. 1221-1230, 2007.

[7] S. Balamurali, P. Jeyadurga, and M. Usha, "Designing of multiple deferred state sampling plan for generalized inverted exponential distribution," Sequential Analysis, vol. 36, no. 1, pp. 76-86, 2017.

[8] A. A. Nadi and B. S. Gildeh, "A group multiple dependent state sampling plan using truncated life test for the Weibull 
distribution," Quality Engineering, vol. 31, no. 4, pp. 1-11, 2019.

[9] M. Aslam, G. S. Rao, and M. Albassam, "Time truncated life tests using the generalized multiple dependent state sampling plans for various life distributions," Statistical Quality Technologies. ICSA Book Series in Statistics, pp. 153-182, Springer, Cham, Switzerland, 2019.

[10] R. Afshari, B. Sadeghpour Gildeh, and M. Sarmad, "Fuzzy multiple deferred state attribute sampling plan in the presence of inspection errors," Journal of Intelligent \& Fuzzy Systems, vol. 33, no. 1, pp. 503-514, 2017.

[11] M. Aslam, M. Azam, and C. H. Jun, "Multiple dependent state sampling plan based on process capability index," Journal of Testing and Evaluation, vol. 41, no. 2, pp. 340-346, 2013.

[12] M. Aslam, M. Azam, and C.-H. Jun, "Multiple dependent state repetitive group sampling plan for Burr XII distribution," Quality Engineering, vol. 28, no. 2, pp. 231-237, 2016.

[13] M. T. Todinov, "Is Weibull distribution the correct model for predicting probability of failure initiated by non-interacting flaws?," International Journal of Solids and Structures, vol. 46, no. 3-4, pp. 887-901, 2009.

[14] C. D. Lai, D. Murthy, and M. Xie, "Weibull distributions and their applications," in Springer Handbook of Engineering Statistics, H. Pham, Ed., Springer, London, England, 2006.

[15] M. Aslam, M. Azam, S. Balamurali, and C. H. Jun, "An economic design of a group sampling plan for a Weibull distribution using a Bayesian approach," Journal of Testing and Evaluation, vol. 43, pp. 1497-1503, 2014.

[16] S. Balamurali, P. Jeyadurga, and M. Usha, "Optimal designing of a multiple deferred state sampling plan for Weibull distributed life time assuring mean life," American Journal of Mathematical and Management Sciences, vol. 36, no. 2, pp. 150-161, 2017.

[17] P. Jeyadurga and S. Balamurali, "Evaluation of an np control chart under truncated life test using quick switching sampling system," Transactions of the Institute of Measurement and Control, vol. 40, no. 12, pp. 3407-3414, 2018.

[18] Z. W. Birnbaum and S. C. Saunders, "A new family of life distributions," Journal of Applied Probability, vol. 6, no. 2, pp. 319-327, 1969.

[19] V. Leiva, C. Marchant, H. Saulo, M. Aslam, and F. Rojas, "Capability indices for Birnbaum-Saunders processes applied to electronic and food industries," Journal of Applied Statistics, vol. 41, no. 9, pp. 1881-1902, 2014.

[20] M. F. Desousa, H. Saulo, V. Leiva, and P. Scalco, "On a tobitBirnbaum-Saunders model with an application to medical data," Journal of Applied Statistics, vol. 45, no. 5, pp. 932-955, 2018.

[21] V. Leiva, F. Ruggeri, H. Saulo, and J. F. Vivanco, "A methodology based on the Birnbaum-Saunders distribution for reliability analysis applied to nano-materials," Reliability Engineering \& System Safety, vol. 157, pp. 192-201, 2017.

[22] N. Balakrishnan, V. Leiva, and J. López, “Acceptance sampling plans from truncated life tests based on the generalized Birnbaum-Saunders distribution," Communications in Statistics - Simulation and Computation, vol. 36, no. 3, pp. 643-656, 2007.

[23] C. Marchant, V. Leiva, F. J. A. Cysneiros, and S. Liu, "Robust multivariate control charts based on Birnbaum-Saunders distributions," Journal of Statistical Computation and Simulation, vol. 88, no. 1, pp. 182-202, 2018.
[24] W. Nelson, "Graphical analysis of accelerated life test data with the inverse power law model," IEEE Transactions on Reliability, vol. R-21, no. 1, pp. 2-11, 1972.

[25] W. G. Ferrell Jr. and A. Chhoker, "Design of economically optimal acceptance sampling plans with inspection error," Computers \& Operations Research, vol. 29, no. 10, pp. 12831300, 2002.

[26] M. S. Fallahnezhad, T. JafariNodoushan, M. S. Owlia, and M. H. Abooie, "Designing an economic repetitive sampling plan in the presence of two markets," IJE Transactions A: Basics, vol. 30, pp. 1017-1028, 2017.

[27] S. Balamurali, P. Jeyadurga, and M. Usha, "Economic design of quick switching sampling system for assuring Weibull distributed mean life," Communications in Statistics - Theory and Methods, vol. 47, no. 2, pp. 385-398, 2018.

[28] L.-F. Hsu and J.-T. Hsu, "Economic design of acceptance sampling plans in a two-stage supply chain," Advances in Decision Sciences, vol. 2012, pp. 1-14, Article ID 359082, 2012.

[29] J. Lieblein and M. Zelen, "Statistical investigation of the fatigue life of deep-groove ball bearings," Journal of Research of the National Bureau of Standards, vol. 57, no. 5, pp. 273-316, 1956. 